\title{
Initiatives, Concepts, and Implementation Practices of FAIR (Findable, Accessible, Interoperable, and Reusable) Data Principles in Health Data Stewardship Practice: Protocol for a Scoping Review
}

Esther Thea Inau ${ }^{1}, \mathrm{MSc}$; Jean Sack ${ }^{2}$, MLS; Dagmar Waltemath ${ }^{1}, \mathrm{PhD}$; Atinkut Alamirrew Zeleke ${ }^{1}, \mathrm{PhD}$

${ }^{1}$ Medical Informatics, Institute for Community Medicine, University Medicine Greifswald, Greifswald, Germany
${ }^{2}$ International Health Department, Johns Hopkins Bloomberg School of Public Health, Baltimore, MD, United States

Corresponding Author:

Esther Thea Inau, MSc

Medical Informatics

Institute for Community Medicine

University Medicine Greifswald

Ellernholzstraße 1-2

Greifswald, 17487

Germany

Phone: 493834867548

Email: inaue@uni-greifswald.de

\section{Abstract}

Background: Data stewardship is an essential driver of research and clinical practice. Data collection, storage, access, sharing, and analytics are dependent on the proper and consistent use of data management principles among the investigators. Since 2016, the FAIR (findable, accessible, interoperable, and reusable) guiding principles for research data management have been resonating in scientific communities. Enabling data to be findable, accessible, interoperable, and reusable is currently believed to strengthen data sharing, reduce duplicated efforts, and move toward harmonization of data from heterogeneous unconnected data silos. FAIR initiatives and implementation trends are rising in different facets of scientific domains. It is important to understand the concepts and implementation practices of the FAIR data principles as applied to human health data by studying the flourishing initiatives and implementation lessons relevant to improved health research, particularly for data sharing during the coronavirus pandemic.

Objective: This paper aims to conduct a scoping review to identify concepts, approaches, implementation experiences, and lessons learned in FAIR initiatives in the health data domain.

Methods: The Arksey and O'Malley stage-based methodological framework for scoping reviews will be used for this review. PubMed, Web of Science, and Google Scholar will be searched to access relevant primary and grey publications. Articles written in English and published from 2014 onwards with FAIR principle concepts or practices in the health domain will be included. Duplication among the 3 data sources will be removed using a reference management software. The articles will then be exported to a systematic review management software. At least two independent authors will review the eligibility of each article based on defined inclusion and exclusion criteria. A pretested charting tool will be used to extract relevant information from the full-text papers. Qualitative thematic synthesis analysis methods will be employed by coding and developing themes. Themes will be derived from the research questions and contents in the included papers.

Results: The results will be reported using the PRISMA-ScR (Preferred Reporting Items for Systematic Reviews and Meta-analyses Extension for Scoping Reviews) reporting guidelines. We anticipate finalizing the manuscript for this work in 2021.

Conclusions: We believe comprehensive information about the FAIR data principles, initiatives, implementation practices, and lessons learned in the FAIRification process in the health domain is paramount to supporting both evidence-based clinical practice and research transparency in the era of big data and open research publishing.

International Registered Report Identifier (IRRID): PRR1-10.2196/22505 


\section{KEYWORDS}

data stewardship; FAIR data principles; health research; PRISMA; scoping review

\section{Introduction}

Advancement in information communication technology is impacting the health ecosystem's technological and analytical capabilities to store, curate, share, and analyze data from standard and nonstandard sources [1]. In the human health domain, big data may be obtained multidimensionally from records in health care facilities, biomedical research institutions, population surveys, surveillance, and patients [2]. Together, professional data management and big data analytics offer high-potential knowledge to transform health care delivery and life sciences research. The availability of data from numerous sources and advanced analytics promise to improve the prevention, diagnosis, and treatment of diseases and the well-being of individuals and societies [3]. However, health data are often stored in independent noncommunicating silos, where open data sharing remains a challenge [4].

Digitalization brings opportunities and concerns in health care data processing. Despite many potential benefits, it also poses potential threats, such as breaches of privacy, disinformation and misinformation, and cyberattacks [5]. There is a need to balance an individual's rights to the protection of personal data from potential threats with the institutions' needs to process these data. The EU General Data Protection Regulation (GDPR) informs this context. The recent reform in the GDPR focuses on the rights and freedoms of people and the establishment of rules for the processing of personal data [6]. The concerns about privacy and personal data protection resulted in reforms of the existing legislation in the European Union. The GDPR aims to reform the existing measures on the topic of personal data protection of EU citizens with a strong input on the rights and freedoms of people and the establishment of rules for the processing of personal data [7]. OpenEHR is a standard that embodies many principles of interoperable and secure software for electronic health records [8].

The European GDPR is the most recent data regulatory framework as of September 2020 and has implications on the ethical sharing of research data [9]. As the EU population continues to be more conscientious about the data protection regulations for citizens' sensitive personal data (eg, EU GDPR), patients and the general public are becoming more aware of the use of their personal data [10]. The principle of data minimization implies that personal data shall be adequate, relevant, and limited to only what is necessary in relation to the purposes for which they are processed [7].

Boeckhout et al [11] highlighted that the GDPR also ensures that the terms of data use, data subjects, and rights in further processing are clearly defined. It has been suggested that FAIR (findable, accessible, interoperable, and reusable) data and metadata standards could help facilitate compliance with the principle of data minimization by allowing for an assessment of which data to reuse based on an analysis of metadata [11].

Beyan et al [12] have shown that an enormous amount of usable health data is currently imprisoned inside the organizational territories of hospitals, clinics, and within patients' devices due to ethical concerns and data protection rules. However, data reuse, even if secondary to data collection and first analysis, may drive more extensive and valuable new research directions than intended for the primary purpose [13]. In Germany, for example, the Medical Informatics Initiative aims to use clinical data to improve health research and facilitate the digitalization of medicine on a national scale [14]. France has also launched the Health Data Hub with similar aims [15]. Currently, researchers and stakeholders are working on infrastructure to support distributed and federated solutions to make the data, software, or digital objects smart in their original silos [12]. Europe would benefit from an integrated infrastructure in which data and computing services for big data can be easily shared and reused, and plans are underway to establish the Europe Research Area for this purpose [16]. Finally, funding agencies and open science advocates are insisting on adherence to open science policies and strategies to manage publicly funded research processes and outcomes [17]. The Health Research Board (HRB) of Ireland, for example, has put in place the HRB Policy on Management and Sharing of Research Data, which requires research to be open. This policy is applicable to data gathered and generated in whole or in part from HRB-funded research, starting from January 1, 2020.

The need for good data stewardship among different stakeholders in scientific research is the basis on which the FAIR data principles (findability, accessibility, interoperability, and reusability) were coined in 2014 by the FORCE11 (The Future of Research Communication and e-Scholarship) community $[18,19]$. These principles were formed to serve as guidance to achieve better research data stewardship practices in the life sciences [20]. They also serve as a set of widely applicable "permissive guidelines," offering a basis for developing flexible community standards for the health data community [21]. Since research papers and data products are now being recognized as key outcomes of the scientific enterprise, various stakeholders in scientific and governmental institutions are increasing their efforts toward establishing more comprehensive plans for data management and stewardship $[16,22]$. Adherence to the FAIR principles has been shown to lead to a more transparent approach to data stewardship, which in turn contributes to the maximal use and reuse of data in the scientific community [23]. Consequently, adherence to the FAIR data principles is more frequently expected by researchers, publishers, funding agencies, and policy makers [24]. Achieving data FAIRness also enhances the discovery of, access to, integration of, and analysis of scholarly and scientific data [25].

In 2020, Vesteghem et al [26] outlined data sharing challenges that make data aggregation costlier and more labor intensive in precision oncology. Obstacles include legal issues that hinder data sharing between research groups, privacy issues, ethical issues, data storage issues, and system incompatibility issues [26]. Various initiatives have been launched to tackle these challenges by standardizing and facilitating the implementation of data pipelines [27,28]. Although the application of the FAIR 
data principles in data stewardship is a fairly new approach in health research, it has been shown to be instrumental in addressing these challenges in the field of precision oncology [14]. It has also been suggested that FAIR data may be useful in addressing the need to generate and share high-quality data to facilitate the World Health Organization elimination goals for neglected tropical diseases [29]. Much work has been conducted to implement the FAIR principles in other domains, such as computational workflows [30], food and nutrition [31], materials science [32], and oceanography [33].

The aims for conducting this work are to (1) provide an overview of applications of the FAIR data principles that are focused on health data research and (2) map out the existing evidence accordingly.

\section{Methods}

\section{Study Framework}

This scoping review will adopt the framework outlined by Arksey and O'Malley [34]. The authors will employ this method to quickly map key concepts underpinning the research area of interest and the main sources and types of evidence available. Our work is focused on an area that we have not seen being reviewed comprehensively. The framework includes the following steps: (1) identifying the research question; (2) identifying relevant studies; (3) selecting the studies; (4) charting the collected data; and (5) collating, summarizing, and reporting the results.

\section{Stage 1: Identifying the Research Questions}

We have already conducted a pilot overview of the existing literature as an informal desk review and literature exploration. This overview included published works in PubMed, Google Scholar, and Web of Science. The medical and public health research librarian used the FAIR data principles' keywords to match medical subject headings (MeSH) used to tag PubMed peer-reviewed literature, along with combinations of terms used in clinical research, public health, health care, pharmacology, and patient data. Multimedia Appendix 1 enumerates the results of these advanced searches.

As part of the ongoing evidence synthesis from medical and human health research journal articles that used FAIR data markup, the bibliographies of key papers were scrutinized for other complementary publications, and those articles were added to the PubMed collections shared with the authors. Further, as the key FAIR data and health articles inspired new citations, often authored by similar consortia of writers or networks of researchers, the newer citing articles were added to the stage 1 collection to demonstrate possible progress in the field of shared or open medical data. Recurrent alerts were set up to capture newly published literature on PubMed, Google Scholar, and Web of Science (Multimedia Appendix 1). White papers, conference publications, guidelines, and other grey literature from the Google and Web of Science alerts were scrutinized and added to a Dropbox of publications for the principal researchers to review. Close examination of key references in bibliographies and citing articles to gauge the impact of FAIR shared data on ensuing research and health practice will be followed as part of the secondary analysis. Publications from 2020 focusing on open sharing of COVID-19 data will be of particular importance in gauging the impact of the FAIR principles on human health data in pandemics.

Our informal desk review has shown that many approaches used in the implementation of the FAIR data principles are applied to the life sciences domain [18]. We have also seen in the literature that there is indeed a growing interest in following the phases of the research life cycle when conducting research $[35,36]$. These findings resonate with the authors' motivation to better understand the approaches used in the implementation of the FAIR data principles and the impact that these implementations may have on the way research in health will be conducted. These findings are also the basis on which the research questions were formulated. As we formulated the research questions, we decided that the review should only include works that show either an actual approach to implementing the FAIR data principles in the health domain or the recorded results of the implementation of the FAIR data principles. The review will exclude works that introduce or give an overview of the FAIR principles. Works that show the implementation of the principles in a domain other than health will also be excluded.

As we intend to conduct this exploratory review in an iterative manner, further refinement of the research questions may become necessary. Close examination of key references in bibliographies and citing articles to gauge the impact of shared data on ensuing research and health practice will be followed as part of the secondary analysis. All proposed refinements of the research questions and search methods will be scrutinized by the authors prior to approval. We will also provide comprehensive provenance information on changes in the protocol to be fully transparent.

\section{Objectives and Research Questions}

The general objective of this protocol is to conduct a scoping review to identify concepts, approaches, implementation experience, and lessons learned from the FAIR data principle initiatives in the health domain. The following research questions (RQs) have been formulated to meet the objective of the scoping review:

- RQ 1: What approaches are being used or piloted in the implementation of the FAIR data principles in the health data domain since the conception of these principles in 2014 ?

- RQ 2: What are the challenges and risks regarding the approaches used in the practical implementation of the FAIR data principles in the health data domain?

- RQ 3: What are the suggested concepts and approaches to mitigating the concerns of the implementation of the FAIR data principles in the health data domain?

- $\quad$ RQ 4: Which are the active public and private research and service networks involved in the implementation of the FAIR data principles in the health data domain?

- RQ 5: What are the reported outcomes for data sharing, data reuse, and research publication after the implementation of the FAIR data principles in the health data domain? 


\section{Stage 2: Identifying Relevant Studies}

With the aid of an experienced research librarian, at least two researchers will identify relevant studies from 3 primary electronic databases: PubMed, Web of Science, and Google Scholar. In addition to those, relevant grey literature from existing networks, relevant organizations, and conferences as well as the reference lists from potential papers will be searched. The keywords for the scoping review search strategies have been categorized tentatively to terms related to the FAIR data principles, data sharing, and health. Although refinement of the selected MeSH terms are possible, open terms have been proposed for the construction of the search strategy of this protocol. The Boolean operators "AND" and "OR" will be used to guide the search strategy. The following descriptors and keywords and their combinations were used to construct the strategies: "open science," "data collection," "data provenance," "open access publishing," "data*," "repositor*," "registr*," "pharma*," "health*," "research," "biomedical research," "data management," "FAIR data principles," "FAIR principles," "FAIR guiding principles," "Data steward*," "Data management systems," "findable," "findability," "access," "accessibility," "interoperable," "interoperability," "reusable," "reusability" (Multimedia Appendix 1).

The PRISMA-ScR (Preferred Reporting Items for Systematic Reviews and Meta-analyses Extension for Scoping Reviews) reporting guidelines will be used for reporting the findings [37]. The operational definition of "health" for this scoping review is based on the European Union's 2018 General Data Protection Regulation and the health ecosystems components framed by the World Health Organization [2,6]. Accordingly, health data in this protocol are defined in the context of data from service and research practice in health services (clinical records, electronic health records and electronic medical records, prescribing, diagnostics, laboratory, insurance, disease surveillance, immunization records, public health reporting, vital statistics, registries, clinical trials, clinical research, and public health research).

As an inclusion criterion, we will consider literature published between January 1, 2014, and December 31, 2020. The start date in 2014 is chosen due to the fact that FAIR concept initiatives and official publications became first available in that year. Moreover, to be included as a potential paper, the literature needs to be published in English and include the scope of FAIR principle applications in the health domain (defined by the operational definition). Literature published before 2014, in a language other than English, and in domain areas other than health or the operational definition of health will be excluded. All search results from online databases and grey literature sources will be exported to a reference management software to eliminate duplications. Unique search results will be exported to a screening tool to facilitate an independent screening process for the potential papers.

\section{Stage 3: Study Selection}

Rayyan software (Qatar Computing Research Institute) has been chosen as the primary screening and data extraction tool to expedite the initial screening of abstracts and titles using a semiautomated process while incorporating a high level of usability. This software supports research teams in the easier exploration of literature searches within a shorter time as well as in sharing and comparing individual researchers' decisions to include or exclude studies [38]. According to the inclusion and exclusion criteria, nonrelevant studies will be excluded from the study at this point. If the relevancy of the publication is unclear from the title or abstract, the reviewer will read the full publication to determine the eligibility of the publication. Any further changes to the search criteria to improve the search findings will be made at this stage as necessary. In the next step, the eligible publications screened in the first stage will be independently read in full by 2 researchers to further determine the relevance of the publication content to the research questions. When agreement cannot be reached during the initial screening and full-text screening stages, an independent researcher will be consulted. A PRISMA flow diagram will be generated to provide visual data for the selection process [37].

\section{Stage 4: Data Charting}

A data-charting form will be used by the reviewers to determine which variables to extract. The form is flexible for continuous updating in an iterative manner during the data-charting process, but any changes will be tracked. The descriptive analytical approach, as described by Arksey and O'Malley [34], will be employed in the data collection process. In this process, the researchers will critically examine the identified articles and documents that meet all of the eligibility criteria and extract the relevant data from each publication using the pretested charting form. The data will be organized into a chart with 2 main sections to describe the overview or summarized basic information of the publication (metadata) and the research questions based on our objectives (Table 1). Initially, 2 authors will independently extract data from the first 5 included studies using the data-charting form and meet to determine whether their approach to data extraction is consistent with the research question and purpose. 
Table 1. Data-charting form.

\begin{tabular}{|c|c|}
\hline Section & Description \\
\hline Section 1: Overview & Summary of the basic information of the publication \\
\hline Publication type & Peer reviewed or grey literature \\
\hline Country & $\begin{array}{l}\text { Name of the country or countries where the study took } \\
\text { place or focused on }\end{array}$ \\
\hline Objective & Aim or objective of the publication \\
\hline Methodology & $\begin{array}{l}\text { The specific procedures or techniques used to identify, se- } \\
\text { lect, process, and analyze information }\end{array}$ \\
\hline Study design and data management & $\begin{array}{l}\text { Includes whether the researchers used quantitative, qualita- } \\
\text { tive, or mixed-method approaches }\end{array}$ \\
\hline Setting of the study & The site in which the researcher conducted the study \\
\hline Summarized results & A short summary of the findings \\
\hline Section 2: Research questions & $\begin{array}{l}\text { Includes the research questions and the date that the litera- } \\
\text { ture was published }\end{array}$ \\
\hline Suggested health care domain-specific FAIRification ${ }^{\mathrm{a}}$ concepts and approaches & $\begin{array}{l}\text { A description of FAIRification concepts and approaches } \\
\text { in the health care domains }\end{array}$ \\
\hline FAIR implementation challenges, risks, and lessons learned & $\begin{array}{l}\text { Encountered challenges or anticipated changes and lessons } \\
\text { learned at different stages of FAIR data principle concept } \\
\text { introduction, infrastructure implementation, and FAIRness } \\
\text { evaluation }\end{array}$ \\
\hline $\begin{array}{l}\text { Active networks involved in the implementation of the FAIR data principles in the } \\
\text { health domain }\end{array}$ & $\begin{array}{l}\text { Dedicated networks of scientific communities, research } \\
\text { institutions, repositories or data archives, consortia, funding } \\
\text { agencies, and citizens who are actively engaged in advocat- } \\
\text { ing FAIR principle data stewardship in the health care do- } \\
\text { mains }\end{array}$ \\
\hline FAIRification reported outcomes & $\begin{array}{l}\text { FAIR implementation outcomes in terms of data sharing, } \\
\text { data reuse, and research publication after imposing FAIR } \\
\text { data principles in health domain }\end{array}$ \\
\hline
\end{tabular}

${ }^{a}$ FAIR: findable, accessible, interoperable, reusable.

\section{Stage 5: Collating, Summarizing, and Reporting the Results}

This scoping review focuses on the range of data curated and the health data research content identified. Quantitative assessment is limited to a count of the number of sources reporting a particular FAIR thematic issue or recommendation. After charting the relevant data from the studies in spreadsheets, the results will be collated and described using summary statistics, charts, figures, and common tools for analytical reinterpretation of the literature [34]. Mapping the themes derived from the research questions (FAIR implementation approaches, available FAIR networks, FAIR infrastructural and security challenges, etc) and other emerging themes during charting and analysis will be done. Moreover, the impact of the findings in relation to the overall study purpose, implications for future research, practice, and policy will be discussed accordingly [34]. The results will be reported using the PRISMA scoping review reporting guidelines [37].

\section{Results}

\section{Overview}

Our PubMed preliminary search has yielded 360 results (Multimedia Appendix 1). The search strategy we used to identify these results will be iteratively revised as we search for the results that best fit the inclusion criteria. We are also working on translating this MeSH search strategy into terms for alerts on the Google Scholar and Web of Science databases. The identification of relevant studies began in April 2020. Data extraction will be carried out in the last quarter of 2020. After completion of steps 1 to 3, we will use the title and abstract and a full-text review to determine the number of studies that meet the inclusion criteria. Full-text data extraction will also be used to confirm the number of studies included. Step 5 will involve summarizing and synthesizing the results. We anticipate finalizing the manuscript for this work by March 2021.

\section{Anticipated Outcomes}

This scoping review will provide insight on the initiatives, concepts, and implementation practices of FAIR data principles in health data stewardship. More specifically, it will allow for the exploration of (1) approaches being used or piloted for the implementation of the FAIR data principles in the health domain since the conception of these principles in 2014; (2) challenges, risks, lessons learned, and the suggested concepts and approaches to mitigate the concerns of implementation of the FAIR data principles in the health domain; (3) active research and service networks involved in the implementation of the FAIR data principles in the health domain; and (4) the reported 
outcomes for data sharing, data reuse, and research publication after the implementation of the FAIR data principles in the health domain. We anticipate increases in data repositories demanding FAIR data markup suitable for artificial intelligence extraction of statistics. We also anticipate a greater demand for the implementation of the FAIR principles in light of the ongoing COVID-19 pandemic as well as more open research activities by public and private research and service networks involved in the implementation of the FAIR data principles in the health domain. An example of such an initiative is the Research Data Alliance [39].

The results will be used to generate recommendations on how to integrate the FAIR principles in health research, and we will generate different knowledge dissemination materials to share project results with various stakeholders, partners, associations, and networks who may benefit from this work.

\section{Discussion}

\section{Future Work}

The findings of this proposed work may be used to help identify the types of available evidence that support the incorporation of FAIR data principles in health. The results will also help to clarify key concepts in the scientific literature and serve as an introduction to how research on FAIR practices is conducted. This methodological framework will help us identify the overall state of research activities that explore initiatives, concepts, and implementation practices of FAIR data principles in health data stewardship. The outcome of this review can be used to further determine areas of research based on current gaps in the literature. Conducting this scoping review will also help determine the practicality and relevance of a full systematic review on the same issues by assessing the availability of literature. Similarly, gaps that still exist in the uptake and implementation of the FAIR principles in health research will also be identified as areas of further research. This work will be of interest to various stakeholders, including health and academic institutions, publishers, researchers, and funding agencies. In the wake of the COVID-19 pandemic, it is extremely critical that health data stewardship is practiced in a FAIR manner to facilitate the globally coordinated response [40]. As this work intends to include works that have been published up until December 31, 2020, we expect that we will gather a lot of information about what has been done worldwide regarding the FAIR data principles in health during this critical time. For purposes of the dissemination of the results of this work, the authors will consider submitting abstracts for presentation to various scientific forums and submit a manuscript for publication in a peer-reviewed journal.

\section{Ethics}

Once complete, this work will be published in a peer-reviewed journal, and the results will also be presented at appropriate forums or conferences. Ethical approval is not required, as only secondary data from published sources will be included in the scoping review and the public is not invited to participate in this work.

\section{Acknowledgments}

We acknowledge support for the article processing charge from the DFG (German Research Foundation; 393148499) and the Open Access Publication Fund of the University of Greifswald.

\section{Conflicts of Interest}

None declared.

\section{Multimedia Appendix 1}

Supplementary Material.

[DOCX File, 20 KB-Multimedia Appendix 1]

\section{References}

1. Khan MA, Uddin MF, Gupta N. Seven V's of Big Data: Understanding Big Data to Extract Value. 2014 Presented at: 2014 Zone 1 Conference of the American Society for Engineering Education; Apr 3-5, 2014; Bridgeport, CT URL: http://www. asee.org/documents/zones/zone1/2014/Professional/PDFs/113.pdf [doi: 10.1109/aseezone1.2014.6820689]

2. Vayena E, Dzenowagis J, Langfeld M. Evolving health data ecosystem. World Health Organization. URL: https://www. who.int/ehealth/resources/ecosystem.pdf [accessed 2020-12-01]

3. McCue ME, McCoy AM. The Scope of Big Data in One Medicine: Unprecedented Opportunities and Challenges. Front Vet Sci 2017;4:194 [FREE Full text] [doi: 10.3389/fvets.2017.00194] [Medline: 29201868]

4. Dash S, Shakyawar SK, Sharma M, Kaushik S. Big data in healthcare: management, analysis and future prospects. J Big Data 2019 Jun 19;6(1):54. [doi: 10.1186/s40537-019-0217-0]

5. McKee M, van Schalkwyk MCI, Stuckler D. The second information revolution: digitalization brings opportunities and concerns for public health. Eur J Public Health 2019 Oct 01;29(Suppl 3):3-6 [FREE Full text] [doi: 10.1093/eurpub/ckz160] [Medline: $\underline{31738440]}$

6. Staunton C, Slokenberga S, Mascalzoni D. The GDPR and the research exemption: considerations on the necessary safeguards for research biobanks. Eur J Hum Genet 2019 Aug;27(8):1159-1167 [FREE Full text] [doi: 10.1038/s41431-019-0386-5] [Medline: $\underline{\text { 30996335] }}$ 
7. Goldsteen A, Ezov G, Shmelkin R, Moffie M, Farkash A. Data minimization for GDPR Compliance in machine learning models. ArXiv. Preprint posted online August 6, 2020 [FREE Full text]

8. Sousa M, Ferreira D, Santos-Pereira C, Bacelar G, Frade S, Pestana O, et al. openEHR Based Systems and the General Data Protection Regulation (GDPR). Stud Health Technol Inform 2018;247:91-95. [Medline: 29677929]

9. Holub P, Kohlmayer F, Prasser F, Mayrhofer MT, Schlünder I, Martin GM, et al. Enhancing Reuse of Data and Biological Material in Medical Research: From FAIR to FAIR-Health. Biopreserv Biobank 2018 Apr;16(2):97-105 [FREE Full text] [doi: 10.1089/bio.2017.0110] [Medline: 29359962]

10. Council of the European Union. Regulation (EU) 2016/679 of the European Parliament and of the Council of 27 April 2016 on the protection of natural persons with regard to the processing of personal data and on the free movement of such data, and repealing Directive 95/46. Off J Eur Union 2016;59(294):1-88 [FREE Full text] [doi: $10.5593 /$ sgemsocial2019v/1.1/s02.022]

11. Boeckhout M, Zielhuis GA, Bredenoord AL. The FAIR guiding principles for data stewardship: fair enough? Eur J Hum Genet 2018 Jul;26(7):931-936 [FREE Full text] [doi: 10.1038/s41431-018-0160-0] [Medline: 29777206]

12. Beyan O, Choudhury A, van Soest J, Kohlbacher O, Zimmermann L, Stenzhorn H, et al. Distributed Analytics on Sensitive Medical Data: The Personal Health Train. Data Intelligence 2020 Jan;2(1-2):96-107 [FREE Full text] [doi: 10.1162/dint_a_00032]

13. European Open Science Cloud. Nat Genet 2016 Jul 27;48(8):821-821. [doi: 10.1038/ng.3642]

14. Semler S, Wissing F, Heyder R. German Medical Informatics Initiative. Methods Inf Med 2018 Jul;57(S 01):e50-e56 [FREE Full text] [doi: 10.3414/ME18-03-0003] [Medline: 30016818]

15. Cuggia M, Combes S. The French Health Data Hub and the German Medical Informatics Initiatives: Two National Projects to Promote Data Sharing in Healthcare. Yearb Med Inform 2019 Aug;28(1):195-202 [FREE Full text] [doi: 10.1055/s-0039-1677917] [Medline: 31419832]

16. Ferrari T, Scardaci D, Andreozzi S. The Open Science Commons for the European Research Area. In: Mathieu PP, Aubrecht C, editors. Earth Observation Open Science and Innovation. Cham, Switzerland: Springer International Publishing; 2018:43-67.

17. Wittenburg P, Sustkova H, Montesanti A. The FAIR Funder pilot programme to make it easy for funders to require and for grantees to produce FAIR Data. ArXiv. Preprint posted online March 6, 2019 [FREE Full text]

18. Wilkinson MD, Dumontier M, Aalbersberg IJ, Appleton G, Axton M, Baak A, et al. The FAIR Guiding Principles for scientific data management and stewardship. Sci Data 2016 Mar 15;3:160018 [FREE Full text] [doi: 10.1038/sdata.2016.18] [Medline: 26978244]

19. Martone M. FORCE11: Building the Future for Research Communications and e-Scholarship. BioScience 2015;65(7):635-635. [doi: 10.1093/biosci/biv095]

20. Hagstrom S. The FAIR data principles. FORCE11. URL: https://www.force11.org/group/fairgroup/fairprinciples [accessed 2020-07-12]

21. Mons B, Neylon C, Velterop J, Dumontier M, da Silva Santos LOB, Wilkinson MD. Cloudy, increasingly FAIR; revisiting the FAIR Data guiding principles for the European Open Science Cloud. ISU 2017 Mar 07;37(1):49-56. [doi: 10.3233/isu-170824]

22. Michener WK. Ten Simple Rules for Creating a Good Data Management Plan. PLoS Comput Biol 2015 Oct 22;11(10):e1004525. [doi: 10.1371/journal.pcbi.1004525] [Medline: 26492633]

23. Boeckhout M, Zielhuis GA, Bredenoord AL. The FAIR guiding principles for data stewardship: fair enough? Eur J Hum Genet 2018 May 17;26(7):931-936. [doi: 10.1038/s41431-018-0160-0] [Medline: 29777206]

24. Jacobsen A, de Miranda Azevedo R, Juty N, Batista D, Coles S, Cornet R, et al. FAIR Principles: Interpretations and Implementation Considerations. Data Intelligence 2020 Jan;2(1-2):10-29. [doi: 10.1162/dint_r_00024]

25. Wise J, de Barron AG, Splendiani A, Balali-Mood B, Vasant D, Little E, et al. Drug Discov Today 2019 Apr;24(4):933-938 [FREE Full text] [doi: 10.1016/j.drudis.2019.01.008] [Medline: 30690198]

26. Vesteghem C, Brøndum RF, Sønderkær M, Sommer M, Schmitz A, Bødker JS, et al. Implementing the FAIR Data Principles in precision oncology: review of supporting initiatives. Brief Bioinform 2020 May 21;21(3):936-945 [FREE Full text] [doi: 10.1093/bib/bbz044] [Medline: $\underline{31263868]}$

27. Kamel PI, Nagy PG. Patient-Centered Radiology with FHIR: an Introduction to the Use of FHIR to Offer Radiology a Clinically Integrated Platform. J Digit Imaging 2018 May 3;31(3):327-333. [doi: 10.1007/s10278-018-0087-6] [Medline: 29725963]

28. Jakob R. [ICD-11-Adapting ICD to the 21st century]. Bundesgesundheitsblatt Gesundheitsforschung Gesundheitsschutz 2018 Jul;61(7):771-777. [doi: 10.1007/s00103-018-2755-6] [Medline: 29869704]

29. Matthews P. FAIR data needed to liberate hepatitis B virus (HBV) from the catch-22 of neglect. J Glob Health 2019 Jun;9(1):010310 [FREE Full text] [doi: 10.7189/jogh.09-010310] [Medline: 30992982]

30. Goble C, Cohen-Boulakia S, Soiland-Reyes S, Garijo D, Gil Y, Crusoe MR, et al. FAIR Computational Workflows. Data Intelligence 2020 Jan;2(1-2):108-121. [doi: 10.1162/dint a 00033]

31. Rychlik M, Zappa G, Añorga L, Belc N, Castanheira I, Donard OFX, et al. Ensuring Food Integrity by Metrology and FAIR Data Principles. Front Chem 2018;6:49 [FREE Full text] [doi: 10.3389/fchem.2018.00049] [Medline: 29872651] 
32. Draxl C, Scheffler M. Big Data-Driven Materials Science and Its FAIR Data Infrastructure. In: Andreoni W, Yip S, editors. Handbook of Materials Modeling. Cham, Switzerland: Springer International Publishing; 2019:1-25.

33. Tanhua T, Pouliquen S, Hausman J, O’Brien K, Bricher P, de Bruin T, et al. Ocean FAIR Data Services. Front Mar Sci 2019 Aug 7;6:440. [doi: 10.3389/fmars.2019.00440]

34. Arksey H, O'Malley L. Scoping studies: towards a methodological framework. Int J Soc Res Methodol 2005 Feb;8(1):19-32. [doi: 10.1080/1364557032000119616]

35. Tang C, Plasek JM, Bates DW. Rethinking Data Sharing at the Dawn of a Health Data Economy: A Viewpoint. J Med Internet Res 2018 Nov 22;20(11):e11519 [FREE Full text] [doi: 10.2196/11519] [Medline: $\underline{30467103}$ ]

36. Fecher B, Friesike S, Hebing M. What Drives Academic Data Sharing? PLoS ONE 2015 Feb 25;10(2):e0118053. [doi: 10.1371/journal.pone.0118053] [Medline: 25714752]

37. Tricco AC, Lillie E, Zarin W, O'Brien KK, Colquhoun H, Levac D, et al. PRISMA Extension for Scoping Reviews (PRISMA-ScR): Checklist and Explanation. Ann Intern Med 2018 Oct 02;169(7):467-473 [FREE Full text] [doi: 10.7326/M18-0850] [Medline: 30178033]

38. Ouzzani M, Hammady H, Fedorowicz Z, Elmagarmid A. Rayyan-a web and mobile app for systematic reviews. Syst Rev 2016 Dec 05;5(1):210 [FREE Full text] [doi: 10.1186/s13643-016-0384-4] [Medline: 27919275]

39. RDA COVID 19 Working Group. RDA COVID19 Case Statement. Research Data Alliance. 2020 Apr 2020. URL: https:/ /www.rd-alliance.org/group/

rda-covid19-rda-covid19-omics-rda-covid19-epidemiology-rda-covid19-clinical-rda-covid19-social [accessed 2020-11-19]

40. Mons B. The VODAN IN: support of a FAIR-based infrastructure for COVID-19. Eur J Hum Genet 2020 May 6;28(6):724-727. [doi: 10.1038/s41431-020-0635-7] [Medline: $\underline{32376989}$ ]

\author{
Abbreviations \\ FAIR: findable, accessible, interoperable, and reusable \\ FORCE11: The Future of Research Communication and e-Scholarship \\ GPDR: General Data Protection Regulation \\ HRB: Health Research Board \\ MeSH: medical subject heading \\ PRISMA-ScR: Preferred Reporting Items for Systematic Reviews and Meta-analyses Extension for Scoping \\ Reviews \\ RQ: research question
}

\author{
Edited by G Eysenbach; submitted 11.09.20; peer-reviewed by L Vergani, C Tang; comments to author 10.10.20; revised version \\ received 02.12.20; accepted 08.12.20; published 02.02.21 \\ Please cite as: \\ Inau ET, Sack J, Waltemath D, Zeleke AA \\ Initiatives, Concepts, and Implementation Practices of FAIR (Findable, Accessible, Interoperable, and Reusable) Data Principles in \\ Health Data Stewardship Practice: Protocol for a Scoping Review \\ JMIR Res Protoc 2021;10(2):e22505 \\ URL: https://www.researchprotocols.org/2021/2/e22505 \\ doi: $10.2196 / 22505$ \\ PMID: $\underline{3528373}$
}

CEsther Thea Inau, Jean Sack, Dagmar Waltemath, Atinkut Alamirrew Zeleke. Originally published in JMIR Research Protocols (http://www.researchprotocols.org), 02.02.2021. This is an open-access article distributed under the terms of the Creative Commons Attribution License (https://creativecommons.org/licenses/by/4.0/), which permits unrestricted use, distribution, and reproduction in any medium, provided the original work, first published in JMIR Research Protocols, is properly cited. The complete bibliographic information, a link to the original publication on http://www.researchprotocols.org, as well as this copyright and license information must be included. 